\title{
QUANTIFYING MINIMAL NON-COLLINEARITY AMONG RANDOM POINTS
}

\author{
IOSIF PINELIS
}

\begin{abstract}
Let $\varphi_{n, K}$ denote the largest angle in all the triangles with vertices among the $n$ points selected at random in a compact convex subset $K$ of $\mathbb{R}^{d}$ with nonempty interior, where $d \geqslant 2$. It is shown that the distribution of the random variable $\lambda_{d}(K) \frac{n^{3}}{3 !}\left(\pi-\varphi_{n, K}\right)^{d-1}$, where $\lambda_{d}(K)$ is a certain positive real number which depends only on the dimension $d$ and the shape of $K$, converges to the standard exponential distribution as $n \rightarrow \infty$. By using the Steiner symmetrization, it is also shown that $\lambda_{d}(K)$ - which is referred to in the paper as the elongation of $K$ - attains its minimum if and only if $K$ is a ball $B^{(d)}$ in $\mathbb{R}^{d}$. Finally, the asymptotics of $\lambda_{d}\left(B^{(d)}\right)$ for large $d$ is determined.
\end{abstract}

\section{CONTEnTs}

1. Summary and discussion

2. Proof of Theorem 1.1

Appendix A. Proof of Proposition 1.2

Appendix B. Proof of Proposition 1.3

References

\section{Summary and discussion}

Let $K$ be a bounded convex subset of $\mathbb{R}^{d}$ with nonempty interior, for some natural $d \geqslant 2$. Let $P_{1}, P_{2}, \ldots$ be random points drawn independently and uniformly from $K$. For each natural $n$, let $T_{n}$ be the set of all subsets of the set $\{1, \ldots, n\}$ of cardinality 3. For each $t=\{i, j, k\} \in T_{n}$, let $X_{t}$ denote the largest angle (with possible angle values in the interval $[0, \pi])$ in the triangle $P_{i} P_{j} P_{k}$ with vertices $P_{i}, P_{j}, P_{k}$. Let

$$
\lambda_{d}(K):=3 \rho_{d} \frac{\mathrm{E}\left|P_{1} P_{2}\right|^{d}}{\mathrm{~V}_{d}(K)}
$$

where

$$
\rho_{d}:=\frac{\pi^{d / 2} \Gamma(d)}{2^{2 d-1} \Gamma((d+1) / 2) \Gamma(d+1 / 2)}
$$

2010 Mathematics Subject Classification. Primary 60C05, 60D05, 60F05; secondary 41A60, 52A22, 52A20, 52A 38, 26B15, 28A75, 05C 38, 62E20, 05C 80.

Key words and phrases. Convex sets, random points, geometric probability theory, integral geometry, maximal angle, convergence in distribution, Steiner symmetrization, asymptotic approximations. 
$\left|P_{1} P_{2}\right|:=\left\|P_{1}-P_{2}\right\|$ is the Euclidean distance between $P_{1}$ and $P_{2}$, and $\mathrm{V}_{d}$ is the $d$-dimensional volume (that is, the Lebesgue measure on $\mathbb{R}^{d}$ ). Let

$$
\varphi_{n, K}:=\max _{t \in T_{n}} X_{t},
$$

the largest angle in all the triangles with vertices among the points $P_{1}, \ldots, P_{n}$.

Theorem 1.1. The random variable (r.v.)

$$
Y_{n, d}:=\lambda_{d}(K) \frac{n^{3}}{3 !}\left(\pi-\varphi_{n, K}\right)^{d-1}
$$

converges as $n \rightarrow \infty$ to an exponential r.v. in distribution:

$$
\mathrm{P}\left(Y_{n, d}>a\right) \rightarrow e^{-a}
$$

for each real $a>0$, and hence uniformly in real $a>0$.

This theorem will be proved in Section 2 ,

In the particular case when $d=2$ and $K$ is a square in $\mathbb{R}^{2}$, a version of Theorem 1.1 with an unspecified constant in place of $\lambda_{d}(K)$ was presented on the MathOverflow site [4].

Note that the constant $\lambda_{d}(K)$ is, naturally, invariant with respect to all translations, homotheties, and orthogonal transformations applied to the set $K$.

The conclusion of Theorem 1.1 can be rewritten as follows:

$$
\mathrm{P}\left(\varphi_{n, K}>c\right)-\left(1-\exp \left\{-\lambda_{d}(K)(\pi-c)^{d-1} \frac{n^{3}}{3 !}\right\}\right) \underset{n \rightarrow \infty}{\longrightarrow} 0
$$

uniformly in $c \in(0, \pi)$. Since $1-\exp \left\{-\lambda_{d}(K)(\pi-c)^{d-1} \frac{n^{3}}{3 !}\right\}$ is increasing in $\lambda_{d}(K)$, we see that, the greater the constant $\lambda_{d}(K)$, the greater the largest angle $\varphi_{n, K}$ tends to be, in an asymptotic stochastic sense.

This provides a reason to refer to the constant $\lambda_{d}(K)$ as the elongation (coefficient) of the convex set $K$. In particular, if the the convex set $K$ is close to a straight line segment, then the volume $\mathrm{V}_{d}(K)$ will be relatively small and hence the elongation $\lambda_{d}(K)$ will be large; accordingly, the largest angle $\varphi_{n, K}$ will then tend to be close to its maximum possible value, $\pi$. Another justification for using this term, elongation, is provided by

Proposition 1.2. Take any natural $d$. The minimum of the elongation $\lambda_{d}(K)$ over all bounded convex subsets $K$ of $\mathbb{R}^{d}$ with nonempty interior is attained only when the closure of $K$ is a closed ball in $\mathbb{R}^{d}$ of a positive radius.

Proposition 1.2 will be proved in Appendix $\mathrm{A}$ The proof is based on the Steiner symmetrization; see e.g. 3]. In particular, we shall show that the Steiner symmetrization does not increase the elongation.

In view of the foregoing discussion, the following appears natural:

Conjecture. The largest angle $\varphi_{n, K}$ becomes stochastically smaller after any Steiner symmetrization of $K$. That is, for any natural $d$, any natural $n \geqslant 3$, any convex subset $K$ of $\mathbb{R}^{d}$, any unit vector $u$ in $\mathbb{R}^{d}$, and any $c \in(0, \pi)$, one has

$$
\mathrm{P}\left(\varphi_{n, \mathrm{~S}_{u} K}>c\right) \leqslant \mathrm{P}\left(\varphi_{n, K}>c\right)
$$

where $\mathrm{S}_{u}$ denotes the Steiner symmetrization along the vector $u$. 
Of course, one may also define the relative elongation of $K$, by dividing the elongation of $K$ by that of the unit ball. Then Proposition 1.2 can be restated as follows: the relative elongation of $K$ is never less than 1, and it equals 1 if and only if the closure of $K$ is a closed ball in $\mathbb{R}^{d}$ of a positive radius.

The asymptotics (for $d \rightarrow \infty$ ) of the elongation coefficient of balls in $\mathbb{R}^{d}$ is presented in

Proposition 1.3. Let $B^{(d)}$ denote the unit ball in $\mathbb{R}^{d}$. Let $P_{1}$ and $P_{2}$ be random points drawn independently and uniformly from $B^{(d)}$. Then

$$
\mathrm{E}\left|P_{1} P_{2}\right|^{d} \sim \frac{1}{\sqrt{6}}\left(\frac{8}{3 \sqrt{3}}\right)^{d} \quad \text { and } \quad \lambda_{d}\left(B^{(d)}\right) \sim \sqrt{3}\left(\frac{2}{3 \sqrt{3}}\right)^{d} \quad \text { as } d \rightarrow \infty .
$$

This proposition will be proved in Appendix B

In particular, it follows from Proposition 1.3 that the $L_{d}$ norm $\left\|\left|P_{1} P_{2}\right|\right\|_{d}:=$ $\left(\mathrm{E}\left|P_{1} P_{2}\right|^{d}\right)^{1 / d}$ of the distance $\left|P_{1} P_{2}\right|$ between the random points $P_{1}$ and $P_{2}$ in the unit ball converges (as $d \rightarrow \infty$ ) to $\frac{8}{3 \sqrt{3}}=1.539 \ldots$, which is strictly less than the diameter 2 of the unit ball. Mainly, this is a consequence of the fact that the maximum of the function $\psi$ in (B.7) occurs at the point $t_{*}=-\frac{1}{3}$.

Let Dlim denote the limit in distribution, for $\operatorname{Dlim}_{n \rightarrow \infty} Y_{n} \stackrel{\mathrm{D}}{=} Y$ to mean that $Y_{n} \underset{n \rightarrow \infty}{\longrightarrow} Y$ in distribution, where $Y$ and the $Y_{n}$ 's are real-valued r.v.'s. Next, let Dlimsup denote the limit superior in distribution, for $\operatorname{Dimsup}_{n \rightarrow \infty} Y_{n} \stackrel{\mathrm{D}}{\leqslant} Y$ to mean that $\limsup _{n \rightarrow \infty} \mathrm{P}\left(Y_{n} \geqslant y\right) \leqslant \mathrm{P}(Y \geqslant y)$ for all real $y$. Similarly, one may let $\operatorname{Dliminf}_{n \rightarrow \infty} Y_{n} \stackrel{\mathrm{D}}{\geqslant} Y$ mean that $\operatorname{Dlimsup}_{n \rightarrow \infty}\left(-Y_{n}\right) \stackrel{\mathrm{D}}{\leqslant}-Y$ or, equivalently, $\liminf _{n \rightarrow \infty} \mathrm{P}\left(Y_{n}>y\right) \geqslant \mathrm{P}(Y>y)$ for all real $y$. Then $\operatorname{Dlim}_{n \rightarrow \infty} Y_{n} \stackrel{\mathrm{D}}{=} Y$ if and only if $\operatorname{Dlimsup}_{n \rightarrow \infty} Y_{n} \stackrel{\mathrm{D}}{\leqslant} Y$ and $\operatorname{Dliminf}_{n \rightarrow \infty} Y_{n} \stackrel{\mathrm{D}}{\geqslant} Y$. In the case when the r.v. $Y$ is actually non-random, here one may simply write $\leqslant, \geqslant$, and $=$ instead of the symbols $\stackrel{\mathrm{D}}{\leqslant}, \stackrel{\mathrm{D}}{\geqslant}$, and $\stackrel{\mathrm{D}}{=}$ (respectively). In these terms, one can now state the following immediate corollary of Theorem 1.1 and Propositions 1.2 and 1.3 .

\section{Corollary 1.4.}

$$
\operatorname{Dlim}_{d \rightarrow \infty} \operatorname{Dlim}_{n \rightarrow \infty}\left(n^{3 /(d-1)}\left(\pi-\varphi_{n, B^{(d)}}\right)\right)=\frac{3 \sqrt{3}}{2} \geqslant \operatorname{Dlimsup}_{d \rightarrow \infty} \operatorname{Dlim}_{n \rightarrow \infty}\left(n^{3 /(d-1)}\left(\pi-\varphi_{n, K_{d}}\right)\right),
$$

where, for each natural $d \geqslant 2, K_{d}$ is an arbitrary bounded convex subset of $\mathbb{R}^{d}$ with nonempty interior.

Note that the double-limit value/bound $\frac{3 \sqrt{3}}{2}$ in (1.6) is non-random; therefore, the symbol D is not overset there. Somewhat crudely but perhaps more transparently, (1.6) may be expressed in terms of an approximate equality $\approx$ and an "approximate inequality" $\lesssim$, as follows: for large $d$ and very large $n$,

$$
\varphi_{n, B^{(d)}} \approx \pi-\frac{3 \sqrt{3} / 2}{n^{3 /(d-1)}} \lesssim \varphi_{n, K_{d}}
$$

with non-random approximate value/bound $\pi-\frac{3 \sqrt{3} / 2}{n^{3 /(d-1)}}$. 


\section{Proof of Theorem 1.1}

Without loss of generality (wlog), (i) the diameter of the set $K$ (defined as $\sup \{\|x-y\|: x, y$ in $K\}$ ) equals 1 and (ii) the point $0 \in \mathbb{R}^{d}$ is in the interior of $K$, so that $B_{0}(r) \subseteq K$ for some real $r>0$; here, as usual, $B_{x}(r):=\left\{y \in \mathbb{R}^{d}:\|x-y\|<r\right\}$ for any $x \in \mathbb{R}^{d}$.

Lemma 2.1. Take any $\delta \in(0,1)$ and any $x \in K_{\delta}$, where

$$
K_{\delta}:=(1-\delta) K:=\{(1-\delta) z: z \in K\} .
$$

Then $B_{x}(r \delta) \subseteq K$.

Proof of Lemma 2.1. Take any $y \in B_{x}(r \delta)$. Then

$$
y=x+(y-x)=(1-\delta) \frac{x}{1-\delta}+\delta \frac{y-x}{\delta} \in K,
$$

because $\frac{x}{1-\delta} \in K, \frac{y-x}{\delta} \in B_{0}(r) \subseteq K$, and the set $K$ is convex. Thus, indeed $B_{x}(r \delta) \subseteq K$.

Take any

$$
\varepsilon \in(0, \pi / 2) .
$$

Take any distinct points $x$ and $y$ in $\mathbb{R}^{d}$, and let

$$
\ell:=\|x-y\| \text {. }
$$

Let $S_{\varepsilon ; x, y}$ denote the set of all points $z \in \mathbb{R}^{d}$ such that the angle $A_{x z y}$ at the vertex $z$ in the triangle $x y z$ is $>\pi-\varepsilon$. In view of the condition $\varepsilon \in(0, \pi / 2)$, one may note that for any $z \in S_{\varepsilon ; x, y}$ the angle $A_{x z y}$ coincides with the largest angle (denoted here by $\left.A_{\{x, y, z\}}\right)$ in the triangle $x y z$. Let

$$
V_{\varepsilon ; d ; x, y}:=\mathrm{V}_{d}\left(S_{\varepsilon ; x, y}\right) .
$$

Lemma 2.2. One has

$$
V_{\varepsilon ; d ; x, y} \sim \rho_{d} \ell^{d} \varepsilon^{d-1},
$$

where $\rho_{d}$ and $\ell$ are as in (1.2) and (2.2).

Here and in what follows, it is assumed by default that the limit relations are as $\varepsilon \downarrow 0$, unless specified otherwise. For any positive expressions $\mathscr{E}_{1}$ and $\mathscr{E}_{2}$, we write $\mathscr{E}_{1} \sim \mathscr{E}_{2}$ if $\mathscr{E}_{1} / \mathscr{E}_{2} \rightarrow 1, \mathscr{E}_{1} \leq \mathscr{E}_{2}$ if $\lim \sup \left(\mathscr{E}_{1} / \mathscr{E}_{2}\right)<\infty$, and $\mathscr{E}_{1}<<\mathscr{E}_{2}$ if $\mathscr{E}_{1} / \mathscr{E}_{2} \rightarrow 0$

Proof of Lemma 2.2. By homothety, wlog $\ell=2$. Note that the angle $A_{x z y}$ equals $\pi-\varepsilon$ if and only if the arc of the circle through the points $x, z, y$ (in this order) is such that the smaller one of the two adjacent angles between the line $x y$ (through the points $x, y)$ and the line tangent to the mentioned arc at the point $x$ equals $\varepsilon$ (of course, the roles of $x$ and $y$ are interchangeable here). It follows that $V_{\varepsilon ; d ; x, y}$ equals twice the volume of the body of revolution obtained by the rotation in $\mathbb{R}^{d}$ of the curvilinear triangle $A E B C D A$ about the line $A C$, where the coordinates of the points $A, B, C, D, E$ in some orthonormal basis of some two-dimensional subspace of $\mathbb{R}^{d}$ are as follows:

$$
\begin{gathered}
A=(\cot \varepsilon, 1), \quad B=(\csc \varepsilon, 0), \quad C=(\cot \varepsilon, 0), \\
D=(\cot \varepsilon, \eta), \quad E=\left(\sqrt{\csc ^{2} \varepsilon-\eta^{2}}, \eta\right),
\end{gathered}
$$


$0<\eta<1$, the curve $A E B$ is the arc of the circle centered at $O=(0,0)$, and the curvilinear triangle $A E B C D A$ is the convex hull of the union of the arc $A E B$ and the singleton set $\{C\}$. This is illustrated in the picture here.

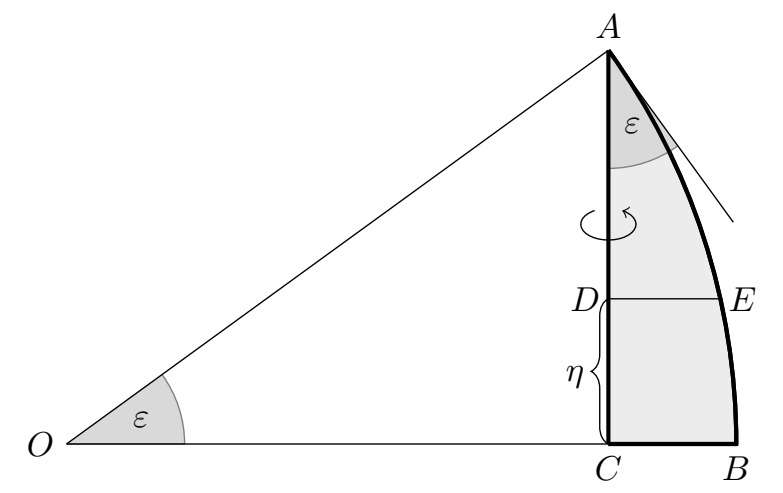

Note that $|C D|=\eta$ and

$$
|D E|=g(\eta):=\sqrt{\csc ^{2} \varepsilon-\eta^{2}}-\cot \varepsilon=\frac{1-\eta^{2}}{\sqrt{\csc ^{2} \varepsilon-\eta^{2}}+\cot \varepsilon} \sim \frac{1}{2}\left(1-\eta^{2}\right) \varepsilon .
$$

So, letting $\beta_{d-1}$ denote the volume of the unit ball in $\mathbb{R}^{d-1}$, we have

$$
V_{\varepsilon ; d ; x, y}=2 \int_{0}^{1} \beta_{d-1} g(\eta)^{d-1} \mathrm{~d} \eta \sim 2 \frac{\beta_{d-1} \varepsilon^{d-1}}{2^{d-1}} \int_{0}^{1}\left(1-\eta^{2}\right)^{d-1} \mathrm{~d} \eta=\rho_{d} 2^{d} \varepsilon^{d-1},
$$

because

$$
\beta_{d-1}=\frac{\pi^{(d-1) / 2}}{\Gamma((d+1) / 2)} \quad \text { and } \quad \int_{0}^{1}\left(1-\eta^{2}\right)^{d-1} \mathrm{~d} \eta=\frac{\sqrt{\pi} \Gamma(d)}{2 \Gamma(d+1 / 2)} .
$$

Thus, Lemma 2.2 is proved.

Lemma 2.3. For the conditional probability that $X_{\{1,2,3\}}>\pi-\varepsilon$ given $P_{1}$ and $P_{2}$, one has

$$
\mathrm{P}\left(X_{\{1,2,3\}}>\pi-\varepsilon \mid P_{1}, P_{2}\right) \lesssim \varepsilon^{d-1} .
$$

Proof of Lemma 2.3. The event $X_{\{1,2,3\}}>\pi-\varepsilon$ implies that

$$
\text { the distance from } P_{3} \text { to the line } P_{1} P_{2} \text { is } \leqslant\left(\left|P_{1} P_{3}\right| \wedge\left|P_{2} P_{3}\right|\right) \sin \varepsilon \leqslant \varepsilon \text {, }
$$

since the diameter of the set $K$ was assumed to equal 1; so, then the point $P_{3}$ lies in the intersection (say $I$ ) of $K$ with (the convex hull of) the round cylinder of radius $\varepsilon$ (with axis $P_{1} P_{2}$ ). The intersection of $I$ with any line parallel to $P_{1} P_{2}$ is a segment of length not exceeding the diameter of $K$, which is 1 . So, $\vee_{d}(I) \leq \varepsilon^{d-1}$, and hence (2.4) follows.

Lemma 2.4. For any $t$ and $s$ in $T_{n}$ such that $t \neq s$ and $t \cap s \neq \emptyset$, one has

$$
\mathrm{P}\left(X_{t}>\pi-\varepsilon, X_{s}>\pi-\varepsilon\right) \lesssim \varepsilon^{2(d-1)},
$$

uniformly over all such s and $t$. 
Proof of Lemma 2.4. Take any $t$ and $s$ in $T_{n}$ such that $t \neq s$ and $t \cap s \neq \emptyset$. Then the cardinality of $t \cap s$ is either 2 or 1 . So, wlog one of the following two cases holds: (i) $t=\{1,2,3\}$ and $s=\{1,2,4\}$ or (ii) $t=\{1,2,3\}$ and $s=\{1,4,5\}$. In case (i),

$$
\begin{aligned}
\mathrm{P}\left(X_{t}>\pi-\varepsilon, X_{s}>\pi-\varepsilon\right) & =\operatorname{EI}\left\{X_{\{1,2,3\}}>\pi-\varepsilon\right\} \mathrm{P}\left(X_{\{1,2,4\}}>\pi-\varepsilon \mid P_{1}, P_{2}, P_{3}\right) \\
& =\operatorname{EI}\left\{X_{\{1,2,3\}}>\pi-\varepsilon\right\} \mathrm{P}\left(X_{\{1,2,4\}}>\pi-\varepsilon \mid P_{1}, P_{2}\right) \\
& \leq \operatorname{EI}\left\{X_{\{1,2,3\}}>\pi-\varepsilon\right\} \varepsilon^{d-1} \\
& =\operatorname{EP}\left(X_{\{1,2,3\}}>\pi-\varepsilon \mid P_{1}, P_{2}\right) \varepsilon^{d-1} \leq \varepsilon^{2(d-1)},
\end{aligned}
$$

by Lemma 2.3 here and elsewhere, I $\{\cdot\}$ is the indicator function. Similarly, in case (ii),

$$
\begin{aligned}
\mathrm{P}\left(X_{t}>\pi-\varepsilon, X_{s}>\pi-\varepsilon\right) & =\mathrm{EI}\left\{X_{\{1,2,3\}}>\pi-\varepsilon\right\} \mathrm{P}\left(X_{\{1,4,5\}}>\pi-\varepsilon \mid P_{1}, P_{2}, P_{3}\right) \\
& =\mathrm{EI}\left\{X_{\{1,2,3\}}>\pi-\varepsilon\right\} \mathrm{P}\left(X_{\{1,4,5\}}>\pi-\varepsilon \mid P_{1}\right) \\
& =\mathrm{EI}\left\{X_{\{1,2,3\}}>\pi-\varepsilon\right\} \mathrm{E}\left(\mathrm{P}\left(X_{\{1,4,5\}}>\pi-\varepsilon \mid P_{1}, P_{4}\right) \mid P_{1}\right) \\
& \leq \mathrm{EI}\left\{X_{\{1,2,3\}}>\pi-\varepsilon\right\} \varepsilon^{d-1} \leq \varepsilon^{2(d-1)} .
\end{aligned}
$$

Lemma 2.4 is now proved.

Lemma 2.5. One has

$$
\mathrm{P}\left(X_{\{1,2,3\}}>\pi-\varepsilon\right) \sim \lambda_{d}(K) \varepsilon^{d-1} .
$$

Proof of Lemma 2.5. The event $\left\{X_{\{1,2,3\}}>\pi-\varepsilon\right\}$ is the union of the events $\left\{A_{P_{1} P_{3} P_{2}}>\pi-\varepsilon\right\},\left\{A_{P_{1} P_{2} P_{3}}>\pi-\varepsilon\right\}$, and $\left\{A_{P_{2} P_{1} P_{3}}>\pi-\varepsilon\right\}$, and these events are pairwise disjoint, in view of (2.1). So,

$$
\mathrm{P}\left(X_{\{1,2,3\}}>\pi-\varepsilon\right)=3 \mathrm{P}\left(A_{P_{1} P_{3} P_{2}}>\pi-\varepsilon\right) .
$$

By Lemma 2.1 with

$$
\delta:=\varepsilon / r,
$$

the event $\left\{\left\{P_{1}, P_{2}\right\} \subseteq K_{\delta}\right\}$ implies $B_{P_{1}}(\varepsilon) \cup B_{P_{2}}(\varepsilon) \subseteq K$. On the other hand, in view of (2.1), the event $\left\{A_{P_{1} P_{3} P_{2}}>\pi-\varepsilon\right\}$ implies that the point on the line $P_{1} P_{2}$ that is the closest one to the $P_{3}$ is between $P_{1}$ and $P_{2}$. Hence, in view (2.5), the event $\left\{A_{P_{1} P_{3} P_{2}}>\pi-\varepsilon,\left\{P_{1}, P_{2}\right\} \subseteq K_{\delta}\right\}$ implies that $P_{3}$ is in the convex hull of the set $B_{P_{1}}(\varepsilon) \cup B_{P_{2}}(\varepsilon)$ and thus in $K$. So, on the event $\left\{\left\{P_{1}, P_{2}\right\} \subseteq K_{\delta}\right\}$,

$$
\mathrm{P}\left(A_{P_{1} P_{3} P_{2}}>\pi-\varepsilon \mid P_{1}, P_{2}\right)=\frac{V_{\varepsilon ; d ; P_{1}, P_{2}}}{\mathrm{~V}_{d}(K)} \sim \rho_{d} \frac{\left|P_{1} P_{2}\right|^{d}}{\mathrm{~V}_{d}(K)} \varepsilon^{d-1}
$$

by Lemma 2.2. whence

$$
\begin{array}{r}
\mathrm{P}\left(A_{P_{1} P_{3} P_{2}}>\pi-\varepsilon,\left\{P_{1}, P_{2}\right\} \subseteq K_{\delta}\right) \sim \rho_{d} \frac{\varepsilon^{d-1}}{\mathrm{~V}_{d}(K)} \mathrm{E}\left|P_{1} P_{2}\right|^{d} \mathrm{I}\left\{\left\{P_{1}, P_{2}\right\} \subseteq K_{\delta}\right\} \\
=\rho_{d} \frac{\varepsilon^{d-1}}{\mathrm{~V}_{d}(K)}(1-\delta)^{3 d} \mathrm{E}\left|P_{1} P_{2}\right|^{d} \sim \rho_{d} \frac{\varepsilon^{d-1}}{\mathrm{~V}_{d}(K)} \mathrm{E}\left|P_{1} P_{2}\right|^{d}
\end{array}
$$


here are details concerning the scaling factor $(1-\delta)^{3 d}$ in the above display: for any $t \in(0,1)$,

$$
\begin{aligned}
\mathrm{E}\left|P_{1} P_{2}\right|^{d} \mathrm{I}\left\{\left\{P_{1}, P_{2}\right\} \subseteq t K\right\} & =\int_{t K} \int_{t K}\left\|x_{1}-x_{2}\right\|^{d} \frac{\mathrm{V}_{d}\left(\mathrm{~d} x_{1}\right)}{\mathrm{V}_{d}(K)} \frac{\mathrm{V}_{d}\left(\mathrm{~d} x_{2}\right)}{\mathrm{V}_{d}(K)} \\
& =\int_{K} \int_{K}\left\|t y_{1}-t y_{2}\right\|^{d} \frac{\mathrm{V}_{d}\left(t \mathrm{~d} y_{1}\right)}{\mathrm{V}_{d}(K)} \frac{\mathrm{V}_{d}\left(t \mathrm{~d} y_{2}\right)}{\mathrm{V}_{d}(K)} \\
& =t^{3 d} \int_{K} \int_{K}\left\|y_{1}-y_{2}\right\|^{d} \frac{\mathrm{V}_{d}\left(\mathrm{~d} y_{1}\right)}{\mathrm{V}_{d}(K)} \frac{\mathrm{V}_{d}\left(\mathrm{~d} y_{2}\right)}{\mathrm{V}_{d}(K)}=t^{3 d} \mathrm{E}\left|P_{1} P_{2}\right|^{d} .
\end{aligned}
$$

Note next that $\mathrm{P}\left(P_{1} \notin K_{\delta}\right)=1-(1-\delta)^{d}<<1$. On the other hand, by Lemma 2.3. $\mathrm{P}\left(A_{P_{1} P_{3} P_{2}}>\pi-\varepsilon \mid P_{1}\right) \leq \varepsilon^{d-1}$. So,

$$
\begin{aligned}
& \mathrm{P}\left(A_{P_{1} P_{3} P_{2}}>\pi-\varepsilon, P_{2} \notin K_{\delta}\right) \\
= & \mathrm{P}\left(A_{P_{1} P_{3} P_{2}}>\pi-\varepsilon, P_{1} \notin K_{\delta}\right) \leq \varepsilon^{d-1} \mathrm{P}\left(P_{1} \notin K_{\delta}\right)<<\varepsilon^{d-1} .
\end{aligned}
$$

Now Lemma 2.5) immediately follows by (2.7), (2.8), (2.9), and (1.1).

To complete the proof of Theorem 1.1, we shall use the key result of Galambos [2], which is in turn based on a combinatorial graph sieve theorem due to Rényi [5]. For readers' convenience, let us restate here the result of [2], as follows.

For each natural $n$, let $T_{n}$ be a set of cardinality $\left|T_{n}\right| \rightarrow \infty$ and let $E_{n}$ be a set of subsets of $T_{n}$ of cardinality 2 such that

$$
\left|E_{n}\right|<<\left|T_{n}\right|^{2} .
$$

In this setting, all limit relations are stated for $n \rightarrow \infty$.

For natural $n$ and $k$ and for $\alpha \in\{0,1\}$, let $H_{n, k}^{(\alpha)}$ denote the set of all subsets $F$ of $T_{n}$ of cardinality $|F|=k$ such that exactly $\alpha$ subsets of $F$ of cardinality 2 belong to $E_{n}$.

Take any real number $a>0$, and let a sequence of real numbers $c_{n}=c_{n}(a)$ be such that the following conditions hold:

$$
\begin{gathered}
\sum_{t \in T_{n}} \mathrm{P}\left(X_{t} \geqslant c_{n}\right) \longrightarrow a ; \\
\sup _{n, t \in T_{n}}\left|T_{n}\right| \mathrm{P}\left(X_{t} \geqslant c_{n}\right)<\infty ; \\
\left|E_{n}\right| \max _{\{t, s\} \in E_{n}} \mathrm{P}\left(X_{t} \geqslant c_{n}, X_{s} \geqslant c_{n}\right) \longrightarrow 0 ;
\end{gathered}
$$

for each natural $k$

$$
\sum_{F \in H_{n, k}^{(0)}}\left[\mathrm{P}\left(\min _{t \in F} X_{t} \geqslant c_{n}\right)-\prod_{t \in F} \mathrm{P}\left(X_{t} \geqslant c_{n}\right)\right] \longrightarrow 0
$$

and for each natural $k$ there is a real number $d_{k}$ such that for all $F \in H_{n, k}^{(1)}$

$$
\mathrm{P}\left(\min _{t \in F} X_{t} \geqslant c_{n}\right) \leqslant d_{k} \mathrm{P}\left(X_{s} \geqslant c_{n}, X_{r} \geqslant c_{n}\right) \prod_{t \in F \backslash\{s, r\}} \mathrm{P}\left(X_{t} \geqslant c_{n}\right),
$$

where $\{s, r\}$ is the only subset of $F$ of cardinality 2 that belongs to $E_{n}$. 
Then

$$
\mathrm{P}\left(\max _{t \in T_{n}} X_{t}<c_{n}\right) \longrightarrow e^{-a} .
$$

(The theorem in [2] was stated in terms of (sub)sequences rather than subsets, but the formulation given above is easily seen to be equivalent to that in 2.)

The four conditions (2.10), (2.13), (2.14), and (2.15) specify a notion of weak dependence of the r.v.'s $X_{t}$. In particular, in the case when the $X_{t}$ 's are independent, it is easy to see that none of these four conditions is needed to deduce (2.16) already from (2.11) and (2.12).

Now we are ready to complete the proof of Theorem 1.1. Indeed, let $T_{n}$ and $X_{t}$ be as described in the beginning of Section 1 so that

$$
\left|T_{n}\right|=\left(\begin{array}{l}
n \\
3
\end{array}\right) \sim \frac{n^{3}}{3 !}
$$

and the r.v.'s $X_{t}$ are exchangeable. Next, let $E_{n}$ be the set of subsets $\{t, s\}$ of $T_{n}$ of cardinality 2 such that $t \cap s \neq \emptyset$.

Then $\left|E_{n}\right| \leq n^{3} \cdot n^{2}$, so that condition (2.10) holds.

Take now indeed any real $a>0$ and let

$$
c_{n}:=c_{n}(a):=\pi-\varepsilon_{n}, \quad \text { where } \quad \varepsilon_{n}:=\left(\frac{a}{\left|T_{n}\right| \lambda_{d}(K)}\right)^{1 /(d-1)},
$$

so that $c_{n} \uparrow \pi$; from now on, all limit relations are stated for $n \rightarrow \infty$. Then conditions (2.11) and (2.12) hold by Lemma 2.5. Condition (2.13) follows by Lemma 2.4 and (2.10). Condition (2.14) is trivial here, because for each $F \in H_{n, k}^{(0)}$ the family of r.v.'s $\left(X_{t}\right)_{t \in F}$ is independent. Finally, condition (2.15) holds (with $d_{k}=1$ ) because for each $F \in H_{n, k}^{(1)}$ and $s, r$ as described in that condition, the family of r.v.'s $\left(X_{t}\right)_{t \in F \backslash\{s, r\}}$ is independent in itself and also independent of the random pair $\left(X_{s}, X_{r}\right)$. (1.3),

Thus, the conclusion (2.16) holds, with $c_{n}$ as in (2.18). In view of (1.4) and

$$
\mathrm{P}\left(\max _{t \in T_{n}} X_{t}<c_{n}\right)=\mathrm{P}\left(Y_{n, d} \frac{\left|T_{n}\right|}{n^{3} / 3 !}>a\right) .
$$

So, to finish the proof of Theorem [1.1, it remains to recall (2.17).

\section{Appendix A. Proof of Proposition 1.2}

As mentioned before, this proof is based on the Steiner symmetrization.

Let $E_{d}(K)$ denote $\mathrm{E}\left|P_{1} P_{2}\right|^{d}$, where, as before, $P_{1}$ and $P_{2}$ are random points drawn independently and uniformly from $K$. Let $\bar{K}$ denote the closure of $K$. Then for any real $\delta>0$ one has $K \subseteq \bar{K} \subseteq(1+\delta) K$, whence

$$
E_{d}(K) \leqslant E_{d}(\bar{K}) \leqslant E_{d}((1+\delta) K)=(1+\delta)^{d} E_{d}(K) \underset{\delta \downarrow 0}{\longrightarrow} E_{d}(K),
$$

so that $E_{d}(\bar{K})=E_{d}(K)$. Similarly, $\vee_{d}(\bar{K})=\mathrm{\vee}_{d}(K)$. So, wlog the bounded convex set $K$ is closed and hence compact, which will be assumed henceforth.

The Steiner symmetrization can be described as follows. Take any unit vector $u \in \mathbb{R}^{d}$. For each $x \in \mathbb{R}^{d}$, there are uniquely determined $\theta(x)=\theta_{u}(x)$ in $\mathbb{R}$ and 
$H(x)=H_{u}(x)$ in the orthogonal complement $\{u\}^{\perp}:=\left\{y \in \mathbb{R}^{d}:\langle y, u\rangle=0\right\}$ of the singleton set $\{u\}$ to $\mathbb{R}$ such that

$$
x=\theta(x) u+H(x)
$$

For each $y \in\{u\}^{\perp}$, let

$$
s(y):=s_{K, u}(y):=\{\alpha \in \mathbb{R}: \alpha u+y \in K\} .
$$

Let then

$$
Y_{u}:=Y_{K, u}:=\left\{y \in\{u\}^{\perp}: s(y) \neq \emptyset\right\} .
$$

Clearly, for each $y \in\{u\}^{\perp}$, the set $s(y)$ is a compact convex subset of $\mathbb{R}$, and this set is nonempty if $y \in \mathscr{Y}_{u}$. So, for each $y \in \mathscr{Y}_{u}$ there exist unique real numbers $a(y)=a_{K}(y)$ and $b(y)=b_{K}(y)$ such that $a(y) \leqslant b(y)$ and

$$
s(y)=[a(y), b(y)] .
$$

Then the Steiner symmetrization, say $\mathrm{S}_{u} K$, of $K$ along the unit vector $u$ is the set

$$
\mathrm{S}_{u} K:=\bigcup_{y \in Y_{K, u}}\left\{\alpha u+y: \frac{a(y)-b(y)}{2} \leqslant \alpha \leqslant \frac{b(y)-a(y)}{2}\right\} .
$$

It is well known and easy to see that $\mathrm{S}_{u} K$ is a compact convex set, of the same volume as $K$ :

$$
\mathrm{V}_{d}\left(\mathrm{~S}_{u} K\right)=\mathrm{V}_{d}(K)
$$

One may also note that that the compact convex set $\mathrm{S}_{u} K$ is determined by the conditions

$$
Y_{\mathrm{S}_{u} K, u}=Y_{K, u} \quad \text { and } \quad s_{\mathrm{S}_{u} K, u}(y)=\left[\frac{a(y)-b(y)}{2}, \frac{b(y)-a(y)}{2}\right]
$$

for all $y \in Y_{K, u}$.

Now we are ready to state

Lemma A.1. $E_{d}\left(\mathrm{~S}_{u} K\right) \leqslant E_{d}(K)$. Moreover, $E_{d}\left(\mathrm{~S}_{u} K\right)=E_{d}(K)$ if and only if $K$ is a ball.

Proof of Lemma A.1. For the random points $P_{1}$ and $P_{2}$ as before and $j \in\{1,2\}$, in view of (A.1) one can write

$$
P_{j}=\xi_{j} u+Q_{j}, \quad \text { where } \xi_{j}:=\theta\left(P_{j}\right) \text { and } Q_{j}:=H\left(P_{j}\right) .
$$

Clearly, the random pairs $\left(\xi_{1}, Q_{1}\right)$ and $\left(\xi_{2}, Q_{2}\right)$ are independent copies of each other. Also, conditionally on $\left(Q_{1}, Q_{2}\right)$, the r.v.'s $\xi_{1}$ and $\xi_{2}$ are independent, and for each $j \in\{1,2\}$ the conditional distribution of $\xi_{j}$ given $\left(Q_{1}, Q_{2}\right)$ is uniform over the interval $\left[A_{j}, B_{j}\right]:=\left[a\left(Q_{j}\right), b\left(Q_{j}\right)\right]$.

Let now $\tilde{P}_{j}, \tilde{\xi}_{j}, \tilde{Q}_{j}, \tilde{A}_{j}, \tilde{B}_{j}$ be defined similarly to $P_{j}, \xi_{j}, Q_{j}, A_{j}, B_{j}$ (respectively), but with $\mathrm{S}_{u} K$ in place of $K$. Note that the random pairs $\left(Q_{1}, Q_{2}\right)$ and $\left(\tilde{Q}_{1}, \tilde{Q}_{2}\right)$ are the same in distribution, and we may and will assume that they are just equal to each other: $\left(\tilde{Q}_{1}, \tilde{Q}_{2}\right)=\left(Q_{1}, Q_{2}\right)$. Then, given $\left(Q_{1}, Q_{2}\right)$, for each $j \in\{1,2\}$ the conditional distribution of $\xi_{j}$ is the same as that of $\tilde{\xi}_{j}+\frac{A_{j}+B_{j}}{2}$. So, letting

$$
\eta:=\xi_{1}-\xi_{2}, \quad \tilde{\eta}:=\tilde{\xi}_{1}-\tilde{\xi}_{2}, \quad C:=\frac{A_{1}+B_{1}}{2}-\frac{A_{2}+B_{2}}{2},
$$

we see that, given $\left(Q_{1}, Q_{2}\right)$, the conditional distribution of $\eta$ is the same as that of $\tilde{\eta}+C$, whereas the conditional distribution of the real valued r.v. $\tilde{\eta}$ given $\left(Q_{1}, Q_{2}\right)$ 
is symmetric (about 0). So, introducing $Z:=\left|Q_{1} Q_{2}\right|=\left\|Q_{1}-Q_{2}\right\|$ and writing the instance $\left|P_{1} P_{2}\right|^{2}=\eta^{2}+Z^{2}$ of the Pythagoras theorem, we have

$$
E_{d}(K)=\mathrm{E} g_{\tilde{\eta}, Z}(|C|) \quad \text { and } \quad E_{d}\left(\mathrm{~S}_{u} K\right)=\mathrm{E} g_{\tilde{\eta}, Z}(0)
$$

where

$$
g_{t, z}(c):=g_{t, z, d}(c):=\frac{1}{2}\left((t+c)^{2}+z^{2}\right)^{d / 2}+\frac{1}{2}\left((-t+c)^{2}+z^{2}\right)^{d / 2} .
$$

for real $t, z, c$. For each $(t, z, d) \in \mathbb{R} \times(0, \infty) \times[1, \infty)$, the function $g_{t, z}=g_{t, z, d}$ is even and strictly convex on $\mathbb{R}$, and hence strictly increasing on $[0, \infty)$. In view of (A.2), this immediately yields the inequality $E_{d}\left(\mathrm{~S}_{u} K\right) \leqslant E_{d}(K)$.

Moreover, it follows that the equality $E_{d}\left(\mathrm{~S}_{u} K\right)=E_{d}(K)$ is possible only if $C=0$ almost surely. By the Fubini theorem, this implies that there is some $y_{1} \in Y_{u}$ such that for almost all $y \in Y_{u}$ one has

$$
\frac{a(y)+b(y)}{2}=\tau_{u}:=\frac{a\left(y_{1}\right)+b\left(y_{1}\right)}{2} ;
$$

the dependence of the functions $a$ and $b$ on the unit vector $u$ (and on the set $K$ ) is implicit here.

Let us now show that A.3 holds for all $y \in Y_{u}$. Toward this end, note first that the set $Y_{u}$ is the orthogonal projection of $K$ onto $\{u\}^{\perp}$, and so, $Y_{u}$ is convex and compact. Moreover, for any $y_{0}$ and $y_{1}$ in $Y_{u}$ and any $t \in(0,1)$ one has $b\left(y_{j}\right) u+y_{j} \in K$ for $j \in\{1,2\}$, whence

$\left[(1-t) b\left(y_{0}\right)+t b\left(y_{1}\right)\right] u+(1-t) y_{0}+t y_{1}=(1-t)\left(b\left(y_{1}\right) u+y_{1}\right)+t\left(b\left(y_{2}\right) u+y_{2}\right) \in K$, which yields $(1-t) b\left(y_{0}\right)+t b\left(y_{1}\right) \in s\left((1-t) y_{0}+t y_{1}\right)$, so that $(1-t) b\left(y_{0}\right)+t b\left(y_{1}\right) \leqslant$ $b\left((1-t) y_{0}+t y_{1}\right)$. This shows that the function $b$ is concave, on $Y_{u}$, and hence continuous on the interior int $Y_{u}$ of $Y_{u}$, by a well-known theorem (see e.g. 6, Theorem 10.1]). It follows that A.3 holds for all $y \in \operatorname{int} Y_{u}$.

Next, take any $y \in Y_{u}$. Take then $x:=b(y) u+y$, so that $x \in K$. By Lemma 2.1, for all $\delta \in(0,1)$ one has int $K \ni(1-\delta) x=(1-\delta) b(y) u+(1-\delta) y$, so that $(1-\delta) y$ is in the orthogonal projection of int $K$ onto $\{u\}^{\perp}$ and hence in int $Y_{u}$. It follows that

$$
\frac{a((1-\delta) y)+b((1-\delta) y)}{2}=\tau_{u},
$$

for all $\delta \in(0,1)$. By the compactness of $K$, there are real $a_{*}$ and $b_{*}$ and a sequence $\left(\delta_{m}\right)$ in $(0,1)$ converging to 0 such that $b\left(\left(1-\delta_{m}\right) y\right) \rightarrow b_{*}$ and $a\left(\left(1-\delta_{m}\right) y\right) \rightarrow a_{*}$, whence $K \ni b\left(\left(1-\delta_{m}\right) y\right) u+\left(1-\delta_{m}\right) y \rightarrow b_{*} u+y$. Therefore and because $K$ is closed, we have $b_{*} u+y \in K$, and so, $b_{*} \leqslant b(y)$. On the other hand, by the concavity of $b$, $b\left(\left(1-\delta_{m}\right) y\right) \geqslant\left(1-\delta_{m}\right) b(y)+\delta_{m} b(0) \rightarrow b(y)$, so that $b_{*}=\lim _{m} b\left(\left(1-\delta_{m}\right) y\right) \geqslant b(y)$. Thus, $b(y)=b_{*}=\lim _{m} b\left(\left(1-\delta_{m}\right) y\right)$ and, similarly, $a(y)=\lim _{m} a\left(\left(1-\delta_{m}\right) y\right)$. Since $\left(1-\delta_{m}\right) y \in \operatorname{int} Y_{u}$ and (A.3) was established for all $y \in \operatorname{int} Y_{u}$, it follows that indeed (A.3) holds for any $y \in Y_{u}$.

This means that the set $K$ is symmertic about the plane

$$
\Pi_{u}:=\left\{x \in \mathbb{R}^{d}:\langle x, u\rangle=\tau_{u}\right\},
$$

for any unit vector $u \in \mathbb{R}^{d}$. By translation, wlog $\bigcap_{i=1}^{d} \Pi_{e_{i}}=\{0\}$, where $\left(e_{1}, \ldots, e_{d}\right)$ is (say) the standard basis of $\mathbb{R}^{d}$. Since $K$ is symmertic about each of the "coordinate" hyperplanes $\Pi_{e_{1}}, \ldots, \Pi_{e_{d}}$, it is easy to see that $K$ is centrally symmetric about the origin. 
So, taking any unit vector $v \in \mathbb{R}^{d}$, one has $a(v)+b(v)=0$. It follows that the hyperplane $\Pi_{v}$ of symmetry of $K$ must pass through the origin. Thus, $K$ is invariant with respect to the reflection in any hyperplane through the origin. On the other hand, by the Cartan-Dieudonné theorem (see e.g. [1]), any orthogonal transformation is the composition of reflections. We conclude that the compact convex set $K$ is invariant with respect to any orthogonal transformation; hence, $K$ is a ball.

To complete the proof of Lemma A.1, it remains to note that, if $K$ is a ball, then obviously $E_{d}\left(\mathrm{~S}_{u} K\right)=E_{d}(K)$.

Lemma A.2. Let $B$ denote the unit ball in $\mathbb{R}^{d}$, and suppose that $\mathrm{V}_{d}(K)=\mathrm{V}_{d}(B)$. Then $E_{d}(K) \geqslant E_{d}(B)$.

Proof of Lemma A.2. Take any $\delta \in(0,1)$. By a well-known result (see cf. 3. Theorem 1.5]), there exist a natural $m$ and unit vectors $u_{1}, \ldots, u_{m}$ in $\mathbb{R}^{d}$ such that

$$
(1+\delta) B \supseteq K_{m}:=\mathrm{S}_{u_{m}} \cdots \mathrm{S}_{u_{1}} K \supseteq(1-\delta) B .
$$

So, in view of Lemma A.1

$$
\begin{aligned}
E_{d}(K) \geqslant E_{d}\left(K_{m}\right) & \geqslant E_{d}((1-\delta) B) \frac{\mathrm{V}_{d}((1-\delta) B)}{\mathrm{V}_{d}\left(K_{m}\right)} \\
& \geqslant E_{d}((1-\delta) B) \frac{\mathrm{V}_{d}((1-\delta) B)}{\mathrm{V}_{d}((1+\delta) B)}=\frac{(1-\delta)^{2 d}}{(1+\delta)^{d}} E_{d}(B),
\end{aligned}
$$

for any $\delta \in(0,1)$. Letting now $\delta \downarrow 0$, one immediately obtains Lemma A.2.

Now it is easy to finish the proof of Proposition 1.2. Indeed, by the scaling properties of $E_{d}(K)=\mathrm{E}\left|P_{1} P_{2}\right|^{d}$ and $\mathrm{V}_{d}(K)$, wlog $\mathrm{V}_{d}(K)=\mathrm{V}_{d}(B)$, where $B$ is the unit ball in $\mathbb{R}^{d}$, as in Lemma A.2. From that lemma and the definition (1.1) of $\lambda_{d}(K)$, it follows immediately that $\lambda_{d}(K) \geqslant \lambda_{d}(B)$.

Suppose now that $\lambda_{d}(K)=\lambda_{d}(B)$. Then $E_{d}(K)=E_{d}(B)$. On the other hand, $E_{d}(K) \geqslant E_{d}\left(\mathrm{~S}_{u} K\right)$ by Lemma A.1 and $E_{d}\left(\mathrm{~S}_{u} K\right) \geqslant E_{d}(B)$ by Lemma A.2 since $\mathrm{V}_{d}\left(\mathrm{~S}_{u} K\right)=\mathrm{V}_{d}(K)=\mathrm{V}_{d}(B)$. Thus, $E_{d}(K) \geqslant E_{d}\left(\mathrm{~S}_{u} K\right) \geqslant E_{d}(B)=E_{d}(K)$, whence $E_{d}\left(\mathrm{~S}_{u} K\right)=E_{d}(K)$, so that, again by Lemma A.1, $K$ is a ball. This completes the proof of Proposition 1.2 .

\section{Appendix B. Proof of Proposition 1.3}

The random pair $\left(P_{1}, P_{2}\right)$ equals $\left(R_{1} U_{1}, R_{2} U_{2}\right)$ in distribution, where $R_{1}$ and $R_{2}$ are real-valued r.v.'s each with density $\mathbb{R} \ni r \mapsto d r^{d-1} \mathrm{I}\{0<r<1\}, U_{1}$ and $U_{2}$ are random vectors each uniformly distributed on the unit sphere, and $R_{1}, U_{1}, R_{2}, U_{2}$ are independent. So, letting $T$ denote the cosine of the angle between the random vectors $U_{1}$ and $U_{2}$, we see that the r.v.'s $R_{1}, R_{2}, T$ are independent, and $\left|P_{1} P_{2}\right|^{2}$ equals $R_{1}^{2}+R_{2}^{2}-2 R_{1} R_{2} T$ in distribution. Moreover - because, by the spherical symmetry, $T$ is independent of (say) $U_{1}$ - the density of $T$ is $\mathbb{R} \ni t \mapsto C_{d}(1-$ $\left.t^{2}\right)^{(d-3) / 2} \mathrm{I}\{-1<t<1\}$, where

$$
C_{d}:=\frac{\Gamma(d / 2)}{\sqrt{\pi} \Gamma((d-1) / 2)} \sim \sqrt{\frac{d}{2 \pi}}
$$

all the limit relations in this proof are of course for $d \rightarrow \infty$. Hence,

$$
\mathrm{E}\left|P_{1} P_{2}\right|^{d}=C_{d} d^{2}\left(J_{d, 1}+J_{d, 2}\right),
$$


where

$$
\begin{gathered}
J_{d, 1}:=\int_{-1}^{0} \Psi_{d}(t)\left(1-t^{2}\right)^{(d-3) / 2} \mathrm{~d} t, \quad J_{d, 2}:=\int_{0}^{1} \Psi_{d}(t)\left(1-t^{2}\right)^{(d-3) / 2} \mathrm{~d} t \\
\Psi_{d}(t):=\int_{0}^{1} \mathrm{~d} r_{1} \int_{0}^{1} \mathrm{~d} r_{2} r_{1}^{d-1} r_{2}^{d-1}\left(r_{1}^{2}+r_{2}^{2}-2 r_{1} r_{2} t\right)^{d / 2} .
\end{gathered}
$$

For $t>0$, the integrand in the integral expression for $\Psi_{d}(t)$ is no greater than $2^{d / 2}$, and so, $\Psi_{d}(t) \leqslant 2^{d / 2}$. Therefore, for $d \geqslant 3$

$$
J_{d, 2} \leqslant 2^{d / 2} \text {. }
$$

Let us now estimate $J_{d, 1}$. Note that

$$
J_{d, 1,1} \leqslant J_{d, 1} \leqslant J_{d, 1,1}+2 J_{d, 1,2}
$$

where

$$
\begin{aligned}
J_{d, 1, j} & :=\int_{-1}^{0} \Psi_{d, j}(t)\left(1-t^{2}\right)^{(d-3) / 2} \mathrm{~d} t \\
\Psi_{d, 1}(t) & :=\int_{1-\delta}^{1} \mathrm{~d} r_{1} \int_{1-\delta}^{1} \mathrm{~d} r_{2} r_{1}^{d-1} r_{2}^{d-1}\left(r_{1}^{2}+r_{2}^{2}-2 r_{1} r_{2} t\right)^{d / 2} \\
\Psi_{d, 2}(t) & :=\int_{0}^{1-\delta} \mathrm{d} r_{1} \int_{0}^{1} \mathrm{~d} r_{2} r_{1}^{d-1} r_{2}^{d-1}\left(r_{1}^{2}+r_{2}^{2}-2 r_{1} r_{2} t\right)^{d / 2} \\
\delta & :=\delta_{d}:=1 / \sqrt{d-1} .
\end{aligned}
$$

For $\left(r_{1}, r_{2}, t\right) \in(0,1-\delta) \times(0,1) \times(-1,0)$, the integrand $r_{1}^{d-1} r_{2}^{d-1}\left(r_{1}^{2}+r_{2}^{2}-\right.$ $\left.2 r_{1} r_{2} t\right)^{d / 2}$ in $\Psi_{d, 2}(t)$ does not exceed

$$
\begin{aligned}
& (1-\delta)^{d-1}\left((1-\delta)^{2}+1-2(1-\delta) t\right)^{d / 2} \\
& \leqslant(1-\delta)^{d-1}(1-t)^{d / 2} 2^{d / 2} \leqslant 2^{d / 2} e^{-\sqrt{d-1}}(1-t)^{d / 2},
\end{aligned}
$$

and so, the latter expression is also an upper bound on $\Psi_{d, 2}(t)$. It follows that

$$
J_{d, 1,2} \leqslant 2^{d / 2} e^{-\sqrt{d-1}} Q_{d}
$$

where

$$
Q_{d}:=\int_{-1}^{0}(1-t)^{d / 2}\left(1-t^{2}\right)^{(d-3) / 2} \mathrm{~d} t .
$$

Letting $v:=1-r_{1}$ and $w:=1-r_{2}$, we have

$$
\begin{aligned}
r_{1}^{2} r_{2}^{2}\left(r_{1}^{2}+r_{2}^{2}-2 r_{1} r_{2} t\right) & =2(1-t)-6(1-t)(v+w)+O\left(v^{2}+w^{2}\right) \\
& =2(1-t)\left[1-3(v+w)+O\left(v^{2}+w^{2}\right)\right] \\
& =2(1-t) \exp \left\{-3(v+w)+O\left(v^{2}+w^{2}\right)\right\} \\
& =2(1-t) \exp \{-(3+o(1))(v+w)\}
\end{aligned}
$$

for $\left(r_{1}, r_{2}, t\right) \in(1-\delta, 1) \times(1-\delta, 1) \times(-1,0)$, whence

$$
\begin{aligned}
\Psi_{d, 1}(t) \sim & 2^{(d-1) / 2}(1-t)^{(d-1) / 2}\left(1^{2}+1^{2}-2 \times 1 \times 1 \times t\right)^{1 / 2} \\
& \times\left(\int_{0}^{\delta} \mathrm{d} v \exp \{-(3+o(1)) v(d-1) / 2\}\right)^{2} \\
& \sim 2^{d / 2}(1-t)^{d / 2} \frac{4}{9 d^{2}}
\end{aligned}
$$


and

$$
J_{d, 1,1} \sim 2^{d / 2} \frac{4}{9 d^{2}} Q_{d}
$$

Comparing this with (B.5), we see that $J_{d, 1,2} \ll J_{d, 1,1}$. Recalling now (B.4), we conclude that

$$
J_{d, 1} \sim J_{d, 1,1} \sim 2^{d / 2} \frac{4}{9 d^{2}} Q_{d}
$$

The needed estimation of $Q_{d}$ is straightforward. Indeed,

$$
Q_{d}=\int_{-1}^{0}(1-t)^{3 / 2} \exp \left\{\frac{d-3}{2} \psi(t)\right\} \mathrm{d} t,
$$

where $\psi(t):=2 \ln (1-t)+\ln (1+t)$. One has $\psi^{\prime}(t)=-\frac{1+3 t}{1-t^{2}} ; \psi^{\prime \prime}(t)=-\frac{1}{(1+t)^{2}}-$ $\frac{2}{(1-t)^{2}}<0 ; \psi^{\prime}(t)=0 \Longleftrightarrow t=t_{*}:=-\frac{1}{3} ; \exp \left\{\psi\left(t_{*}\right)\right\}=\frac{32}{27} ;$ and $\psi^{\prime \prime}\left(t_{*}\right)=-\frac{27}{8}$. So, standard reasoning yields

$$
\begin{aligned}
Q_{d} & \sim \int_{-1}^{0}\left(1-t_{*}\right)^{3 / 2} \exp \left\{\frac{d-3}{2}\left[\psi\left(t_{*}\right)+\frac{1}{2} \psi^{\prime \prime}\left(t_{*}\right)\left(t-t_{*}\right)^{2}\right]\right\} \mathrm{d} t \\
& \sim\left(1-t_{*}\right)^{3 / 2} \exp \left\{\frac{d-3}{2} \psi\left(t_{*}\right)\right\} \frac{\sqrt{2 \pi}}{\sqrt{-\psi^{\prime \prime}\left(t_{*}\right) d / 2}}=\sqrt{\pi} \frac{3 \sqrt{3}}{4 \sqrt{d}}\left(\frac{32}{27}\right)^{d / 2} .
\end{aligned}
$$

Hence, by (B.6),

$$
J_{d, 1} \sim 2^{d / 2} \frac{4}{9 d^{2}} \sqrt{\pi} \frac{3 \sqrt{3}}{4 \sqrt{d}}\left(\frac{32}{27}\right)^{d / 2}=\frac{\sqrt{\pi}}{d^{2} \sqrt{3 d}}\left(\frac{64}{27}\right)^{d / 2} .
$$

Comparing this with (B.3), we see that $J_{d, 2}<<J_{d, 1}$. Now (B.2) and (B.1) yield

$$
\mathrm{E}\left|P_{1} P_{2}\right|^{d} \sim C_{d} d^{2} J_{d, 1} \sim C_{d} \frac{\sqrt{\pi}}{\sqrt{3 d}}\left(\frac{64}{27}\right)^{d / 2} \sim \frac{1}{\sqrt{6}}\left(\frac{8}{3 \sqrt{3}}\right)^{d},
$$

which proves the first asymptotic equivalence in (1.5). The second asymptotic equivalence there now follows immediately by (1.1), (1.2), the first equality in (2.3) (with $d-1$ replaced by $d$ ), and the asymptotic equivalence $\Gamma(\alpha+1 / 2) \sim \sqrt{\alpha} \Gamma(\alpha)$ as $\alpha \rightarrow \infty$.

\section{References}

[1] C. Fuller. A constructive proof of the Cartan-Dieudonné-Scherk theorem in the real or complex case. J. Pure Appl. Algebra, 215(5):1116-1126, 2011.

[2] J. Galambos. On the distribution of the maximum of random variables. Ann. Math. Statist., 43:516-521, 1972.

[3] B. Klartag. Rate of convergence of geometric symmetrizations. Geom. Funct. Anal., 14(6):1322-1338, 2004.

[4] I. Pinelis. Alignment of random points. MathOverflow, http://mathoverflow.net/q/247328, 2016.

[5] A. Rényi. A general method to prove theorems of probability and some of its applications (in Hungarian). Magyar Tud. Akad. Mat. Fiz. Oszt. Kozl., 11:79-105, 1961.

[6] R. T. Rockafellar. Convex analysis. Princeton Landmarks in Mathematics. Princeton University Press, Princeton, NJ, 1997. Reprint of the 1970 original, Princeton Paperbacks.

Department of Mathematical Sciences, Michigan Technological University, HoughTON, Michigan 49931, USA, E-Mail: IPINELis@Mtu.EDU 